\title{
MODELING OF A SYSTEM WITH HYBRID DYNAMICS
}

\author{
Vratislav HLADKÝ, L'uboš POPOVIČ, Ján SARNOVSKÝ \\ Department of Cybernetics and Artificial Intelligence, Faculty of Electrical Engineering and Informatics, \\ Technical University of Košice, Letná 9, 04200 Košice, Slovak Republic, tel.: +421 55602 4216, \\ e-mail: vratislav.hladky@tuke.sk, lubos.popovic@tuke.sk, jan.sarnovsky@tuke.sk
}

\begin{abstract}
The article deals with modeling of a system with hybrid dynamics and with two tools for modeling of such systems. For purpose of modeling we chose the system of coupled tanks. The tanks are situated in different heights and dynamics of the system changes in the moment when the liquid level in the second tank runs over the height of bottom of the first tank. That represents hybrid nature of this system. We chose two tools for simulation of hybrid systems - MPT toolbox and HYSDEL and we specifically aim on modeling of the system of coupled tanks by both these tools and on comparison of these tools. The tools are free however they do not work independently but as a part of the simulation language Matlab.
\end{abstract}

Keywords: hybrid systems, simulation, Matlab, MPT toolbox, HYSDEL

\section{INTRODUCTION}

The systems which have been traditionally studied by automatic control theory involve mainly familiar dynamical systems that are governed by ordinary differential or difference equations. On the other hand there are many situations in which such models are not appropriate. For example consider hybrid dynamic systems in which the state of systems includes both continuous and discrete variables (robotic systems, rulebased controllers, fault-tolerant systems, systems with many failure modes, etc.). The control of these systems involves discrete sets of alternative interpretations of observed data, models for each of these alternatives and optimal decision. This higher level discrete decision is then incorporated into a feedback control policy. Such a high-level controller should be able to deal with situations which may involve decision processes. In control practice it is quite common to use several different controllers and to switch among them with some type of logical device. Some examples are systems with selectors, which have been used for constraint control for a long time, systems with gain scheduling, etc. These systems are commonly used for control of chemical processes, power stations and in flight control. Other examples of systems with mode switching are found in robotics or in the expert controller with a hierarchical structure where a collection of controllers are juggled by an expert system. All these systems are hybrid control systems $[1,4]$.

One definition of the hybrid control system is: the hybrid control system is a control system where the plant or the controller contains discrete modes that together with continuous equations govern the behavior of the system [5]. This general definition covers basically every existing control system.

Lately there has been a considerable effort in trying to unify some of the approaches and get a more general theory for hybrid systems. The fundamental problem with hybrid systems is their complex mixture of discrete and continuous variables. Such systems are very general and they have appeared in many different domains. They have, for example, attracted much interest in control as well as in computer science. In automatic control the focus has been on the continuous behavior, while computer science has emphasized the discrete aspects.

It is generally difficult to get analytical solutions to mixed difference/differential equations. For some problems it is possible to do qualitative analysis for aggregated models. Because of the lack of good analysis methods, many investigations of hybrid systems have relied on simulation [1,5]. Therefore we decided to choose two concrete modeling tools for hybrid systems - MPT toolbox and HYSDEL and to model a chosen hybrid system by these tools.

\section{SYSTEMS WITH HYBRID DYNAMICS}

Today complex systems have hybrid character thus in general we can say that these systems involve continuous and discrete variables. Especially existence of both types of variables gives the hybrid nature to such systems. Evolution of the hybrid systems we can so describe by equations that include logical, discrete and continuous variables. Continuous dynamics of these systems is mostly described by equation with continuous or discrete time. Discrete dynamics is mostly described by digital automaton or input - output transition system with a finite number of states [1].

Further we will mainly deal with the hybrid systems by which the continuous dynamics is generally described by linear differential equations and the discrete dynamics by so called state automatons and both are synchronized by the same clock pulse. Such systems are often called as discrete hybrid automatons.

The specific example of the discrete hybrid automaton is represented by piecewise affinne systems (PWA systems) that were mentioned first time in [9]. Actual mode of these systems depends on actual position of the state vector in specific area only. Both mentioned hybrid dynamic systems are essentially equivalent and therefore the systems described as discrete hybrid automatons can be transformed into piecewise affinne systems [11].

From point of view of our simulation the most important part of the discrete hybrid automaton is 
switched affine system. Switched affine system is a set of linear affine systems [12]:

$$
\begin{aligned}
& x_{r}(k+1)=A_{i(k)} x_{r}(k)+B_{i(k)} u_{r}(k)+f_{i(k)} \\
& y_{r}(k)=C_{i(k)} x_{r}(k)+D_{i(k)} u_{r}(k)+g_{i(k)}
\end{aligned}
$$

where $k \in Z^{+}$is the time indicator, $x_{r} \in X_{r} \subseteq R^{n}$ is the continuous state vector, $u_{r} \in U_{r} \subseteq R^{m}$ is the continuous input vector, $y_{r} \in Y_{r} \subseteq R^{p}$ is the continuous output vector, $\left\{A_{i}, B_{i}, f_{i}, C_{i}, D_{i}, g_{i}\right\}_{i \in I}$ is a set of matrices of suitable dimensions, and the mode $i(k) \in I$ ( $I$ is the set of integers) is an input signal that chooses one of the linear dynamics of the system.

A switched affine system given by equation (1) preserves the value of the state when a switch occurs. A switched affine system can be rewritten as the combination of linear terms and if-then-else rules. The equation (1) is then equivalent to the form [12]:

$$
\begin{aligned}
& z_{1}(k)= \begin{cases}A_{1} x_{r}(k)+B_{1} u_{r}(k)+f_{1} ; & \text { if }(i(k)=1) \\
0 ; & \text { otherwise }\end{cases} \\
& z_{s}(k)= \begin{cases}A_{s} x_{r}(k)+B_{s} u_{r}(k)+f_{s} ; & \text { if }(i(k)=s) \\
0 ; & \text { otherwise }\end{cases} \\
& x_{r}(k+1)=\sum_{i=1}^{s} z_{i}(k)
\end{aligned}
$$

where $z_{i}(k) \in R^{n}, i=1, \ldots, s$. Similarly we can also transform the equation (2).

Other parts of discrete hybrid automaton are: event generator - is a mathematical object that generates a logic signal according to the satisfaction of a linear affine constraint of the system, finite state machine (or automaton) - is a discrete dynamic process that evolves according to a logic state function and in principle it controls mode selector, mode selector - it defines the actual mode of the system [12].

\section{MODELING OF THE SYSTEM OF COUPLED TANKS}

As an example of the hybrid system we chose the system of coupled tanks which is shown in Fig. 1 [2]. But for our purposes we have modified this system - the first tank is spherical. The dynamic behaviour of the system changes in time and it is described by several sets of differential equations. At specified time periods it switches among these sets of differential equations and this feature represents hybrid character of the system.

The parameters of the tank are: $R_{1}=1,2 \mathrm{~m}, F_{2}=3,72$ $\mathrm{m}^{2}, k_{11}=4 \mathrm{lit}^{2,5} \mathrm{~s}^{-1}, k_{22}=3,56 \mathrm{lit}^{2,5} \mathrm{~s}^{-1}, h=1,35 \mathrm{~m}$. The tanks are situated in different heights (see Fig. 1) and dynamics of the system changes in the moment when the liquid level in the second tank runs over the height of bottom of the first tank. Mathematical description changes

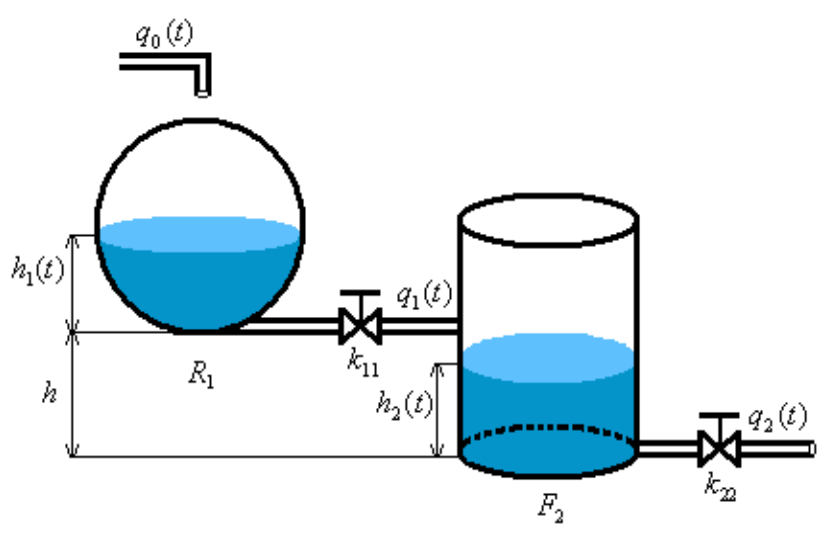

Fig. 1 The system of coupled tanks

from the description of the system without interaction to the description of the system with interaction at this moment. Therefore it is useful to obtain two separate mathematical descriptions - for the system without interaction and for the system with interaction.

These mathematical models are produced by equation of materials balance for mass of the liquid which is in the form (verbal description): 'sum of mass flow on input' = 'sum of mass flow on output' + 'speed of accumulation of mass in the system' $[2,8]$.

At first we formulate the dynamic model of the system without interaction. In this case the level of liquid in the second tank does not run over the height of the bottom of the first tank. For the first tank we have:

$\rho q_{0}(t)=\rho q_{1}(t)+\rho F_{1}(t) \frac{d h_{1}(t)}{d t}$

where: $q_{0}(t)$ - volume flow on the input, $q_{1}(t)$ - volume flow on the output, $F_{1}(t)$ - variable sectional area of the first tank, $h_{1}(t)$ - liquid level in the first tank, $\rho-$ consistency of liquid.

We assume that consistency of liquid $\rho$ is constant, so equation (6) is in the form:

$q_{0}(t)=q_{1}(t)+F_{1}(t) \frac{d h_{1}(t)}{d t}$

The sectional area of the first tank is variable and it can be formulated by equation:

$$
F_{1}(t)=\pi\left(2 R_{1} h_{1}(t)-h_{1}^{2}(t)\right)
$$

where: $R_{1}$ - radius of the first tank.

Following Bernoulli equation the flow $q_{1}(t)$ is the function of the liquid level in the first tank by the form:

$q_{1}(t)=f_{1} \sqrt{2 g h_{1}(t)}$

where: $f_{1}$ - sectional area of efflux nozzle of the first tank, $g-$ acceleration of gravity.

By mergence of constants we have:

$q_{1}(t)=k_{11} \sqrt{h_{1}(t)}$ 
Substituting $q_{1}(t)$ from equation (10) and $F_{1}(t)$ from equation (8) to equation (7) we have:

$q_{0}(t)=k_{11} \sqrt{h_{1}(t)}+\pi\left(2 R_{1} h_{1}(t)-h_{1}^{2}(t)\right) \frac{d h_{1}(t)}{d t}$

The liquid level $h_{1}$ represents the first state variable.

Similarly for the second tank we can obtain:

$k_{11} \sqrt{h_{1}(t)}=k_{22} \sqrt{h_{2}(t)}+F_{2} \frac{d h_{2}(t)}{d t}$

$k_{22}=f_{2} \sqrt{2 g}$

where: $h_{2}(t)$ - liquid level in the second tank, $F_{2}-$ sectional area of the second tank, $f_{2}$ - sectional area of efflux nozzle of the second tank. We assume that sectional area of the second tank $F_{2}$ is constant.

Equations (11) and (12) represent the dynamic model of the system of coupled tanks without interaction.

For simulation of the system it is important to determine the liquid levels in steady state. If the input flow is constant then the liquid levels in the tanks are fixed and their derivations are equal to zero:

$\frac{d h_{1}^{s}}{d t}=0 ; \quad \frac{d h_{2}^{s}}{d t}=0$

where: $h_{1}{ }^{s}$ - liquid level in the first tank in steady state, $h_{2}{ }^{s}-$ liquid level in the second tank in steady state.

Then the model of the first tank in steady state is in the form:

$q_{0}^{s}=q_{1}^{s} \quad$ or $\quad q_{0}^{s}=k_{11} \sqrt{h_{1}^{s}}$

From this equation we can simply calculate the liquid level in the steady state. Similarly we can calculate the steady state of the liquid level for the second tank.

Further similarly as for the system without interaction we can obtain mathematical model of the dynamic system of coupled tanks with interaction. The equation for the first tank is in the form [7]:

$$
\begin{aligned}
& q_{0}(t)= \\
& =\operatorname{sign}\left(h_{1}(t)-\left(h_{2}(t)-h\right)\right) k_{11} \sqrt{\left|h_{1}(t)-\left(h_{2}(t)-h\right)\right|}+ \\
& +\pi\left(2 R_{1} h_{1}(t)-h_{1}^{2}(t)\right) \frac{d h_{1}(t)}{d t}
\end{aligned}
$$

and for the second tank:

$$
\begin{aligned}
& \operatorname{sign}\left(h_{1}(t)-\left(h_{2}(t)-h\right)\right) k_{11} \sqrt{\left|h_{1}(t)-\left(h_{2}(t)-h\right)\right|}= \\
& =F_{2} \frac{d h_{2}(t)}{d t}+k_{22} \sqrt{h_{2}(t)}
\end{aligned}
$$

Enumeration of the liquid level in the steady state is identical as for the first tank.

For the purposes of modeling of this hybrid system in HYSDEL we have to linearize both mathematical model of the system of coupled tanks - without interaction and with interaction. We will realize the linearization for the first tank. The equation (11) is nonlinear with regard to $h_{1}$. At first we subtract equation describing the system in the steady state from equation describing the same dynamic system:

$$
q_{0}(t)-q_{0}^{s}=k_{11} \sqrt{h_{1}(t)}-k_{11} \sqrt{h_{1}^{s}}+F_{1}^{s} \frac{d\left(h_{1}(t)-h_{1}^{s}\right)}{d t}
$$

where:

$$
F_{1}^{s}=\pi\left(2 R_{1} h_{1}^{s}-\left(h_{1}^{s}\right)^{2}\right)
$$

Because we perform lizearization in small area of operating point (steady state) we can consider the sectional area of the first tank $F_{1}$ as constant (19).

It is useful to define deviation values:

$$
\Delta h_{1}(t)=h_{1}(t)-h_{1}^{s} ; \quad \Delta q_{0}(t)=q_{0}(t)-q_{0}^{s}
$$

We expand nonlinear item $\sqrt{h_{1}(t)}$ into the Taylor series and we neglect higher terms of the series:

$\sqrt{h_{1}(t)}=\sqrt{h_{1}^{s}}+\left.\frac{\partial\left(\sqrt{h_{1}(t)}\right)}{\partial h_{1}}\right|_{h_{1}^{s}}\left(h_{1}(t)-h_{1}^{s}\right)=$

$=\sqrt{h_{1}^{s}}+\frac{1}{2 \sqrt{h_{1}^{s}}}\left(h_{1}(t)-h_{1}^{s}\right)$

Substituting to the equation (18) we obtain:

$$
\begin{aligned}
& q_{0}(t)-q_{0}^{s}=k_{11} \sqrt{h_{1}^{s}}+k_{11} \frac{1}{2 \sqrt{h_{1}^{s}}}\left(h_{1}(t)-h_{1}^{s}\right)- \\
& -k_{11} \sqrt{h_{1}^{s}}+F_{1}^{s} \frac{d\left(h_{1}(t)-h_{1}^{s}\right)}{d t}
\end{aligned}
$$

For the sake of simplicity we define the following constant:

$$
k_{1}=\frac{k_{11}}{2 \sqrt{h_{1}^{s}}}
$$
form:

Then the linearized model of the first tank is in the

$$
\Delta q_{0}(t)=k_{1} \Delta h_{1}(t)+F_{1}^{s} \frac{d \Delta h_{1}(t)}{d t}
$$

The linearized mathematical model for the second tank and for the system with interaction we can obtain by identical way as described above.

\subsection{Modeling of the system of coupled tanks by MPT toolbox}

It is very useful to model and simulate the hybrid system shown in Fig. 1 by utilization of MPT toolbox. 
This toolbox is free software and it is possible to download it from the web page: http://control.ee.ethz.ch/ $\sim$ mpt/downloads/. The toolbox does not work independently but as a part of the simulation language Matlab. By MPT toolbox we are able to model directly equations describing a dynamic system, in our case equations (11), (12), (16) and (17). In addition the MPT toolbox enables to rewrite piecewise affine systems to Matlab. It is only required differential equations that represent the hybrid dynamic of the system and condition / conditions by which the dynamics of the system changes. At creating the model in Matlab we produce S-functions that it is possible to use in Simulink too. The differential equations that represent particular dynamics are for purposes of writing to S-function of the MPT toolbox in following form.

1. The system without interaction:

$$
\begin{aligned}
& \frac{d h_{1}(t)}{d t}=\frac{q_{0}(t)}{\pi\left(2 R_{1} h_{1}(t)-h_{1}^{2}(t)\right)}-\frac{k_{11} \sqrt{h_{1}(t)}}{\pi\left(2 R_{1} h_{1}(t)-h_{1}^{2}(t)\right)} \\
& \frac{d h_{2}(t)}{d t}=\frac{k_{11} \sqrt{h_{1}(t)}}{F_{2}}-\frac{k_{22} \sqrt{h_{2}(t)}}{F_{2}}
\end{aligned}
$$

2. The system with interaction:

$$
\begin{aligned}
& \frac{d h_{1}(t)}{d t}=\frac{q_{0}(t)}{\pi\left(2 R_{1} h_{1}(t)-h_{1}^{2}(t)\right)}- \\
& -\frac{\operatorname{sign}\left(h_{1}(t)-\left(h_{2}(t)-h\right)\right) k_{11} \sqrt{\left|h_{1}(t)-\left(h_{2}(t)-h\right)\right|}}{\pi\left(2 R_{1} h_{1}(t)-h_{1}^{2}(t)\right)}
\end{aligned}
$$

$\frac{d h_{2}(t)}{d t}=$

$=\frac{\operatorname{sign}\left(h_{1}(t)-\left(h_{2}(t)-h\right)\right) k_{11} \sqrt{\left|h_{1}(t)-\left(h_{2}(t)-h\right)\right|}}{F_{2}}-$

$-\frac{k_{22} \sqrt{h_{2}(t)}}{F_{2}}$ form:

The condition of change of the dynamics is in the

$$
h_{2}(t)>h
$$

The trajectory of the liquid level $h_{2}(t)$ and process of change of the system dynamics that we obtained by simulation of the system by MPT toolbox are shown in Fig. 2.

\subsection{Modeling of the system of coupled tanks by HYSDEL}

Simulation of the same hybrid system (the system of coupled tanks) by utilization of the tool HYSDEL will be described in this part. HYSDEL is abbreviation from English term HYbrid System DEscription Language. This toolbox is also free software and it is possible to download
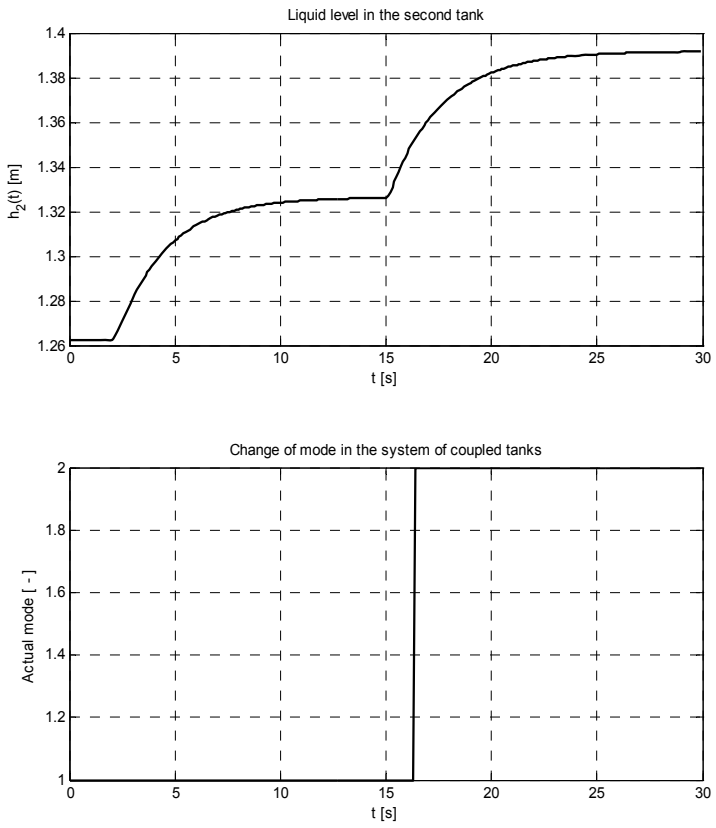

Fig. 2 The time responses of the liquid level $h_{2}(t)$ and the process of mode change - simulation by MPT toolbox

it from the web page: http://control.ee.ethz.ch/ hybrid/ hysdel/hysdel.php. It does not work independently but as a part of the simulation language Matlab. HYSDEL has been developed in Italian - Swiss cooperation and it is also commercially used by ABB company in Switzerland [12]. Preffered application of the HYSDEL is in the area of modeling of discrete hybrid automata [11].

Source code of HYSDEL consists of two main parts that are INTERFACE and IMPLEMENTATION, and syntax of this language is based on the principle of $\mathrm{C}$ language.

The part INTERFACE contains declarations and it is distributed to another parts called STATE, INPUT, OUTPUT and PARAMETER, where sequence of particular parts is not fundamental [12]. For declaration of a real variable we use the key word REAL, for example "REAL variable_name [min, $\max ]$ " where $\min$ and $\max$ are limits of given variable. For declaration of a Boolean variable it is used the key word BOOL.

The part IMPLEMENTATION consists of another special parts which described relations within defined variables. The special parts will be described in following [12]:

- OUTPUT - allows specifying static linear and logic relations for the output vector of the system.

- $\mathrm{AD}$ - allows to define Boolean variables from continuous ones, and is based exactly on the same semantics of the event generator, which is detailly described in [12].

- $\quad$ LOGIC - allows to specify arbitrary functions of Boolean variables: In particular the mode selector is a Boolean function and therefore it can be modeled in this section.

- DA - defines continuous variables according to if-then-else conditions on Boolean variables. This section models part of the switched affine system. 
- CONTINUOUS - describes the linear dynamics, expressed as difference equations. This section models the remaining third equation of the switched affine system.

- $\quad$ LINEAR - allows also to define a continuous variable as an affine function of continuous variables.

- AUTOMATA - specifies the state transition equations of the finite state machine as Boolean functions.

- $\quad$ MUST - specifies constraints on continuous and Boolean variables, i.e., linear constraints and Boolean formulas.

Need for linearization of dynamic models of systems with sequential discretization of obtained differential equations is a certain disadvantage of this language for description of the hybrid systems. After this manner processed equations will be in the form:

1. System without interaction:

$\Delta h_{1}(t+T)=\left(1-\frac{k_{1}}{\frac{F_{1}^{s}}{T}}\right) \Delta h_{1}(t)+\frac{\Delta q_{0}(t)}{\frac{F_{1}^{s}}{T}}$

$\Delta h_{2}(t+T)=\frac{k_{1}}{\frac{F_{2}}{T}} \Delta h_{1}(t)+\left(1-\frac{k_{2}}{\frac{F_{2}}{T}}\right) \Delta h_{2}(t)$

2. System with interaction:

$\Delta h_{1}(t+T)=\left(1-\frac{k_{1}}{\frac{F_{1}^{s}}{T}}\right) \Delta h_{1}(t)+\frac{k_{1}}{\frac{F_{1}^{s}}{T}} \Delta h_{2}(t)+\frac{\Delta q_{0}(t)}{\frac{F_{1}^{s}}{T}}$

$\Delta h_{2}(t+T)=\frac{k_{1}}{\frac{F_{1}^{s}}{T}} \Delta h_{1}(t)-\left(\frac{k_{1}}{\frac{F_{2}}{T}}+\frac{k_{2}}{\frac{F_{2}}{T}}-1\right) \Delta h_{2}(t)$

Condition of change of the dynamics from the system without interaction to the system with interaction or reversely is again defined by form (29).

The trajectory of the liquid level $h_{2}(t)$ and process of change of the system dynamics that we obtained by simulation of the system by HYSDEL are shown in Fig. 3.

\section{CONCLUSIONS}

We have successfully simulated the system with hybrid dynamics by two special tools for simulation of such systems. The hybrid nature of the system consists in interaction between tanks, especially in fact that the connecting pipe is not at the bottom of the second tank but in height $h$. We have also modeled directly equations (25), (26), (27) and (28) by block in Simulink for purposes of
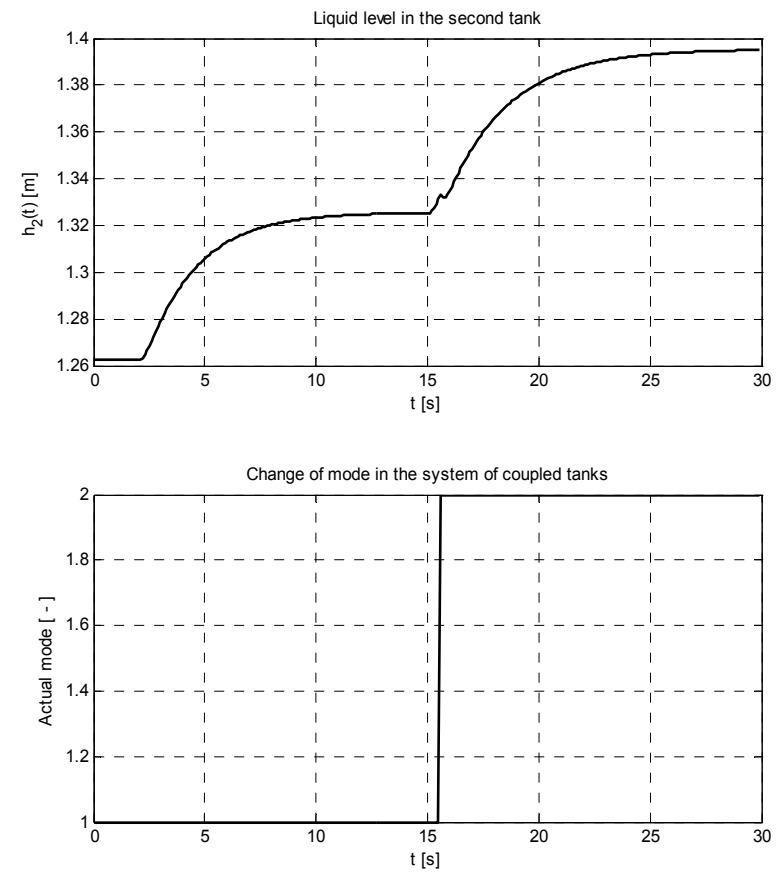

Fig. 3 The time responses of the liquid level $h_{2}(t)$ and the process of mode change - simulation by HYSDEL

comparison of trajectories of liquid levels modeled by MPT toolbox and HYSDEL. Following experiences from modeling of the hybrid system we can say that the results of simulations by MPT toolbox and by HYSDEL are equivalent but simulation of a hybrid system by MPT toolbox is more simple and comfortable than by HYSDEL. By MPT toolbox we are able to simulate directly equations describing a system without additional modifications of the equations. For modeling in HYSDEL we have to linearize the mathematical model of the system. In this case it can be quite difficult to determine operating point and also to determine switch condition. For that reason HYSDEL appears to be more suitable for modeling such systems where states of the system are switched by a switch device. In our case there is no real switching device, it means that we do not use any logical variables. The dynamic of the system of coupled tanks is continuous and at the moment when the liquid level in the second tank $h_{2}(t)$ reaches the height $h$ it changes only mathematical model of the system from the system without interaction to the system with interaction, and it represents the actual mode of the system.

\section{ACKNOWLEDGMENTS}

This work is the result of the project implementation: Center of Information and Communication Technologies for Knowledge Systems (ITMS project code: 26220120020) supported by the Research \& Development Operational Program funded by the ERDF.

\section{REFERENCES}

[1] BRANICKY, M.: Studies in Hybrid Systems: Modeling, Analysis, and Control. Ph.D. diss., Massachussetts Institute of Technology, 1995. 
[2] HIRMAJER, T. - FIKAR, M.: Optimal Control of Systems with Hybrid Dynamics, AT\&P Journal, vol. 8, no. 12 , pp. 81-84, 2005.

[3] HIRMAJER, T. - FIKAR, M.: Dynamic Optimization of a Hybrid Coupled Tanks System, Journal of Electrical Engineering, vol. 57, no. 3, pp. 167-172, 2006.

[4] HLADKÝ, V. - SARNOVSKÝ, J.: Hybrid Control of Large Scale Systems, Proceedings of the $13^{\text {th }}$ International Conference on Process Control, High Tatras, Slovak Republic, CD ROM, June 11-14, 2001.

[5] MALMBORG, J.: Analysis and Design of Hybrid Control Systems. Ph.D. diss., Lund Institute of Technology, 1998.

[6] POPOVIČ, L'. - HLADKÝ, V. - SARNOVSKÝ, J.: Modeling and Control of Laboratory Model Ball\&Plate by MPT Toolbox, Proceedings of International Conference Cybernetics and Informatics, Vyšná Boca, Slovak Republic, CD ROM, February 10-13, 2010.

[7] NOSKIEVIČ, P.: Modeling and Identification of Systems, Ostrava, Montanex, 1999, pp. 56-70.

[8] SARNOVSKÝ, J. - HLADKÝ, V. - JADLOVSKÁ, A: Control of Large-scale Systems, Košice, Elfa, 2005.

[9] SONTAG, E. D.: Nonlinear regulation: The piecewise linear approach, IEEE Transactions Automatic Control, vol. 26, no. 2, pp. 346-358, 1981.

[10] TORISI, F. D. - BEMPORAD, A.: Discrete-time Hybrid Modeling and Verification, Proceedings of $40^{\text {th }}$ IEEE Conference on Decision and Control, Orlando, Florida, pp. 2899-2904, 2001.

[11] TORISI, F. D. - BEMPORAD, A.: HYSDEL A Tool for Generating Computational Hybrid Models, IEEE Transactions Control Systems Technology, vol. 12, no. 2, pp. 235-249, 2004.
[12] TORISI, F. D. - BEMPORAD, A. - BERTINI, G. HERTACH, P. - JOST, D. - MIGNONE, D.: HYSDEL 2.0.5 - user's manual, pp. 35, 2002.

Received November 10, 2010, accepted March 11, 2011

\section{BIOGRAPHIES}

Vratislav Hladký was born on 12.5.1968. In 1991 he graduated (MSc) at the Department of Cybernetics and Artificial Intelligence of the Faculty of Electrical Engineering and Informatics at Technical University in Košice. He defended his $\mathrm{PhD}$ in the field of Control engineering and automation in 1996. His scientific research is focusing on dynamic and control of complex and hybrid systems.

L’uboš Popovič was born on 5.6.1984. In 2007 he graduated (MSc) at the Department of Cybernetics and Artificial Intelligence of the Faculty of Electrical Engineering and Informatics at Technical University in Košice. Since 2007 he is a PhD student at the Department of Cybernetics and Artificial Intelligence. His $\mathrm{PhD}$ thesis in the field of Cybernetics; is "Principle of requisite variety in control systems". His scientific research is focusing on dynamic and control of complex and hybrid systems.

Ján Sarnovský was born on 23.3.1945. He received his MSc. in the field of Automation at Electrotechnical faculty of Slovak Technical University in Bratislava in 1968. In. 1980 he defended his doctoral candidate thesis and obtained scientific degree CSc. He obtained Professor of Technical Cybernetics in 1993. From 1989 he is a head of Department of Cybernetics and Artificial Intelligence of the Faculty of Electrical Engineering and Informatics at Technical University in Košice. He is coordinator of some national scientific grants. His scientific research is focusing on control of dynamic systems and multi-agent systems. 